\title{
Effect of position on lumbar lordosis in patients with adult spinal deformity
}

\author{
Tatsuya Yasuda, MD, ${ }^{1}$ Tomohiko Hasegawa, MD, PhD, ${ }^{2}$ Yu Yamato, MD, PhD, ${ }^{2}$ \\ Daisuke Togawa, MD, PhD, ${ }^{2,3}$ Sho Kobayashi, MD, PhD, ${ }^{2}$ Go Yoshida, MD, PhD, ${ }^{2}$ \\ Tomohiro Banno, MD, PhD, ${ }^{2}$ Hideyuki Arima, MD, PhD, ${ }^{2}$ Shin Oe, MD, ${ }^{2,3}$ and \\ Yukihiro Matsuyama, MD, $\mathrm{PhD}^{2}$
}

'Department of Orthopaedic Surgery, Hamamatsu Medical Center; ${ }^{2}$ Department of Orthopaedic Surgery, Hamamatsu University of Medicine; and 'Division of Geriatric Musculoskeletal Health, Hamamatsu University School of Medicine, Hamamatsu, Japan

OBJECTIVE The purpose of this study was to evaluate the effect of position on lumbar lordosis (LL) in adult spinal deformity (ASD) patients.

METHODS The authors evaluated the radiographic data of ASD patients who underwent posterior corrective fusion surgery from the thoracic spine to $L 5, S 1$, or the ilium for the treatment of ASD of the lumbar spine. The spinopelvic parameters were measured in the standing position preoperatively. LL was also evaluated in the supine position preoperatively and in the prone position on the surgical frame. Changes in LL were compared between groups.

RESULTS Eighty-five patients were included. The average LL in standing, supine, and prone positions was $11.8^{\circ}, 24.3^{\circ}$, and $24.0^{\circ}$, respectively. $\mathrm{LL}$ increased significantly from standing to supine or prone position $(p<0.001)$. In 80 patients (94.1\%), the difference between supine $\mathrm{LL}$ and prone $\mathrm{LL}$ was within $5^{\circ}$. Change in $\mathrm{LL}$ from standing to prone position was significantly higher in the severe deformity group.

CONCLUSIONS The lordotic effect of intraoperative prone positioning was remarkable in patients with severe deformities. LL in the supine position was approximately the same as that in the prone position. Therefore, assessing preoperative supine lateral lumbar radiographs enables one to plan corrective spinal surgeries in ASD patients.

https://thejns.org/doi/abs/10.3171/2018.3.SPINE1879

KEYWORDS lumbar lordosis; adult spinal deformity; prone position; corrective spinal surgery

$\mathrm{R}$ ECENT studies have shown that sagittal spinopelvic alignment, including pelvic position, plays a critical role in the health-related quality of life of patients with adult spinal deformity (ASD) ${ }^{4,7,12,13}$ Therefore, the goal of corrective surgery for ASD is to achieve and maintain optimal spinopelvic alignment. Lumbar lordosis (LL) is one of the important parameters for optimal sagittal alignment. The achievement of adequate LL is necessary for good results in corrective surgeries in spinal fusion. Additionally, LL is one of the main parameters that can be controlled by surgeons. Thus, some formulae have been developed to calculate the degree of optimal LL in corrective surgeries for ASD. ${ }^{11,16}$

There are some correction techniques to restore LL, such as posterior column osteotomy, pedicle subtraction osteotomy, and vertebral column resection. These osteotomy techniques have varying correcting power to achieve lordosis. Posterior column osteotomy provides about only $10^{\circ}$ of correction per level, but it can be used at multiple levels. Pedicle subtraction osteotomy and vertebral column resection provide about $30^{\circ}$ or more correction per level. ${ }^{1,2}$ Optimal LL can be achieved through a combination of these techniques depending on the type of deformity.

Usually, spinopelvic parameters including LL are evaluated on standing radiographs. Thus, the correction angle that can be achieved by osteotomy is evaluated by radiographs taken in the standing position. However, since LL may be different depending on position, preoperative surgical planning for deformity correction should be based on intraoperative prone position. Although various authors

ABBREVIATIONS ASD = adult spinal deformity; LL = lumbar lordosis; PI-LL = pelvic incidence minus lumbar lordosis; $\mathrm{PT}$ = pelvic tilt; SVA = sagittal vertical axis; $\mathrm{TK}$ = thoracic kyphosis.

SUBMITTED January 19, 2018. ACCEPTED March 29, 2018.

INCLUDE WHEN CITING Published online August 3, 2018; DOI: 10.3171/2018.3.SPINE1879. 


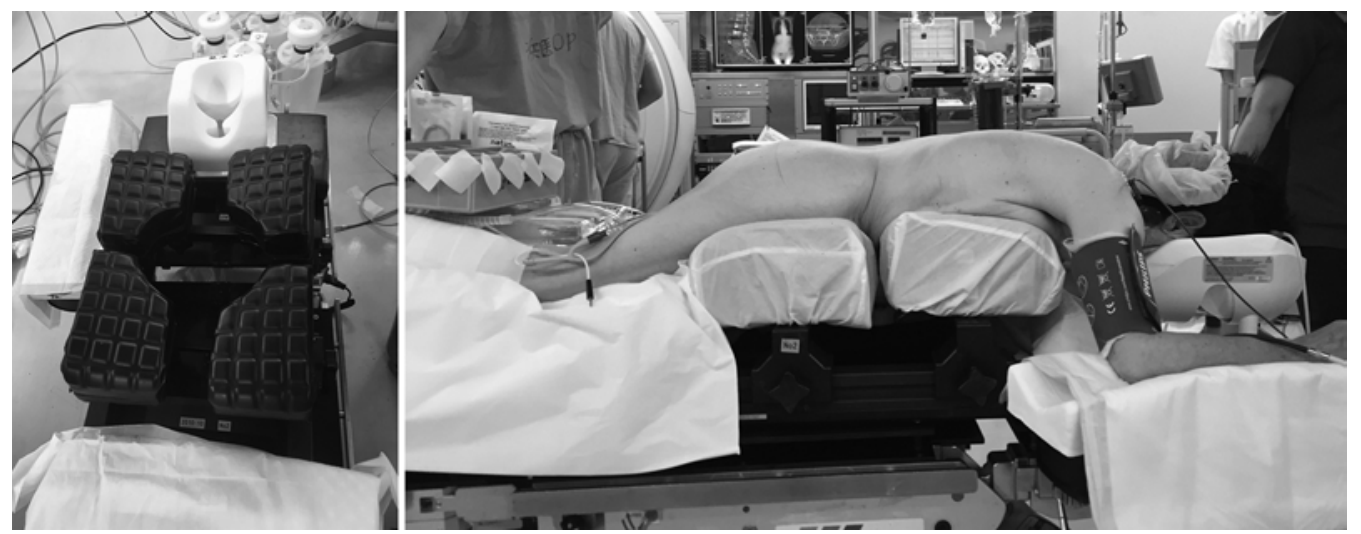

FIG. 1. Left: The spinal frame image viewed from the top of the table. Right: Intraoperative prone position. Patients were positioned on the frame with hips and knees in slight flexion. The spinal frame consisted of 2 chest pads and 2 anterior pelvic pads.

have analyzed the effects of operative positioning on LL during surgeries for lumbar degenerative disease, $, 5,8-10,14,15$ there are few reports about the impact of positioning on the change in LL in patients with ASD.

The purpose of this study was to investigate the impact of positioning on LL in patients with ASD to help with preoperative planning.

\section{Methods}

\section{Patient Sample}

Our study group consisted of adult patients who underwent posterior corrective fusion surgery from the thoracic spine to L5, S1, or the ilium for the treatment of ASD of the lumbar spine. The study protocol was approved by the institutional review board of Hamamatsu University School of Medicine, Shizuoka, Japan. Inclusion criteria for ASD were patients aged 40 years or older and the presence of at least one of the following measures of spinal deformity: coronal Cobb angle of $20^{\circ}$ or greater, sagittal vertical axis (SVA) of $5 \mathrm{~cm}$ or more, pelvic tilt (PT) of $25^{\circ}$ or greater, and thoracic kyphosis (TK) of $60^{\circ}$ or greater.

The etiologies of ASD were as follows: degenerative kyphoscoliosis in 44 patients, degenerative kyphosis in 19 , kyphosis with vertebral fracture in 9, neuromuscular deformity in 10 , and adult idiopathic deformity in 3 . Two patients had a history of spinal fusion (one level and two levels, respectively).

\section{Radiographic Measurements}

Standing radiographic evaluation was performed according to an established positioning protocol for obtaining lateral slot scan digital standing radiographs ${ }^{6}$ preoperatively. Radiographic parameters included SVA, L1-S1 LL, T5-12 thoracic kyphosis (TK), pelvic tilt (PT), and pelvic incidence minus lumbar lordosis (PI-LL) by using digitized radiographs of the entire lateral spine in the standing position. LL was also evaluated in supine and prone positions. Supine LL was measured using lateral lumbar radiographs taken in the supine position preoperatively. Additionally, prone LL was measured using lateral lumbar radiographs of the spinal frame that were obtained in the prone position after induction of general anesthesia just before beginning the surgery. The spinal frame had 2 chest pads and 2 anterior pelvic pads (Fig. 1 left). All patients were positioned on the frame with hips and knees in slight flexion (Fig. 1 right). All radiographs were measured by 7 board-certified spine surgeons using the Synapse application available in the Fujifilm PACS application suite (Fujifilm Holdings).

\section{Impact of Positioning}

Change in LL in each position was analyzed. Additionally, correlation between the change in LL and spinopelvic parameters was evaluated using Spearman's correlation.

The patients were divided into 2 groups depending on preoperative PI-LL: a severe deformity group (PI-LL $>30^{\circ}$ ) and a mild deformity group (PI-LL $\leq 30^{\circ}$ ). The Mann-Whitney U-test was used to identify significant differences between the groups.

\section{Results}

\section{Relevant Characteristics of the Study Group}

Eighty-five patients met our inclusion criteria; there were 11 men and 74 women whose mean age was 70.2 years.

\section{Radiographic Parameters of Spinopelvic Alignment}

Radiographic parameters of spinal alignment are summarized in Table 1. The average SVA improved from $127 \mathrm{~mm}$ to $45 \mathrm{~mm}$ after surgery, while the average TK

TABLE 1. Spinopelvic radiographic parameters in standing position

\begin{tabular}{lc}
\hline Parameter & Preoperative Standing Radiograph \\
\hline SVA $(\mathrm{mm})$ & $127.0 \pm 89.8$ \\
\hline $\mathrm{LL}\left({ }^{\circ}\right)$ & $7.8 \pm 23.0$ \\
\hline $\mathrm{TK}\left({ }^{\circ}\right)$ & $25.0 \pm 17.6$ \\
\hline $\mathrm{PT}\left({ }^{\circ}\right)$ & $35.5 \pm 11.7$ \\
\hline $\mathrm{PI}-\mathrm{LL}\left({ }^{\circ}\right)$ & $43.4 \pm 23.9$ \\
\hline
\end{tabular}




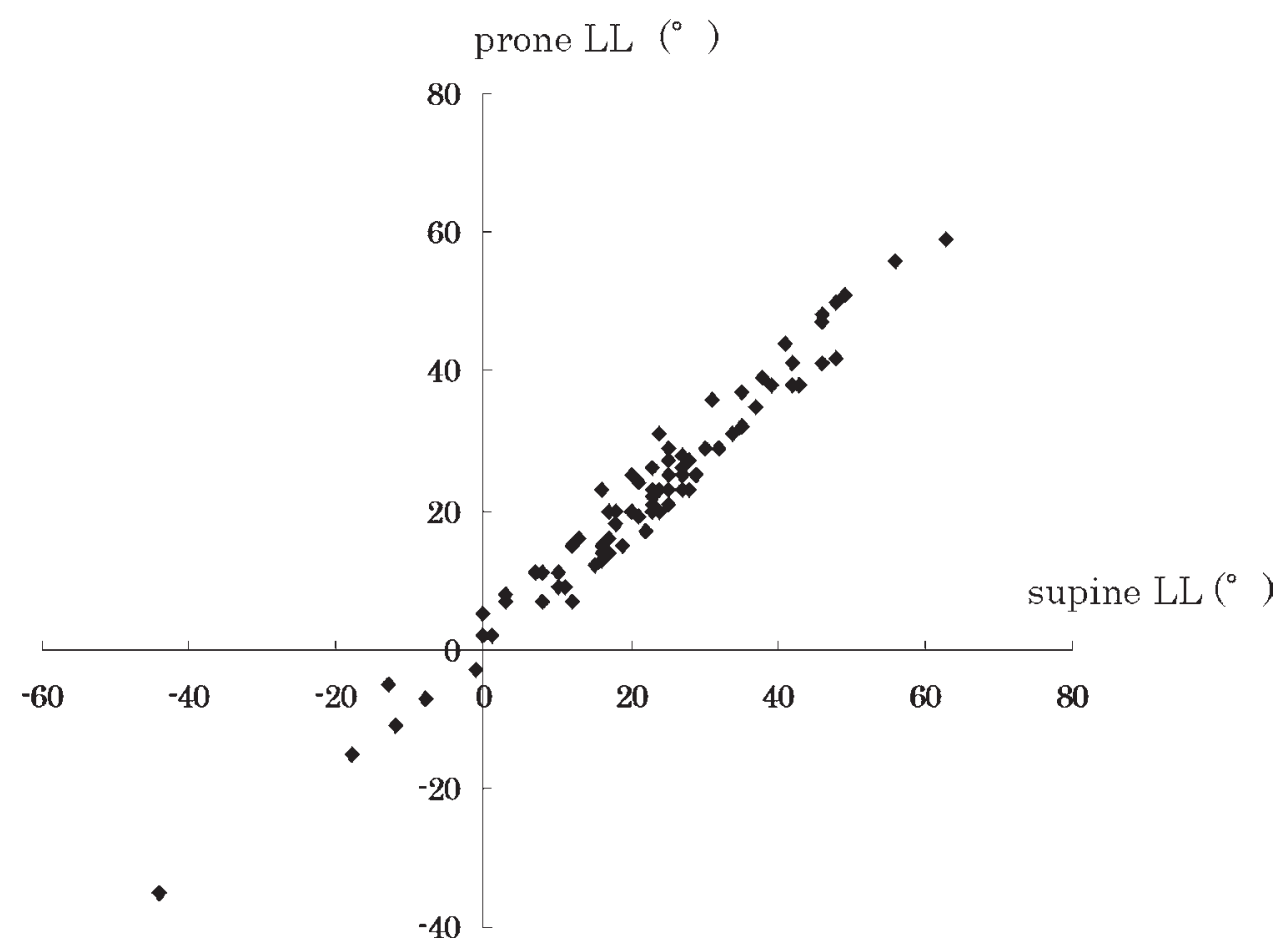

FIG. 2. Scatter plots of $L L$ in the supine position and prone position. $L L$ in the supine position was nearly the same as that in the prone position.

increased from $25.0^{\circ}$ to $34.8^{\circ}$ and the anteversion in PT improved from $35.5^{\circ}$ to $23.9^{\circ}$. The average LL increased from $7.8^{\circ}$ to $40.0^{\circ}$, and PI-LL improved from $43.4^{\circ}$ to $12.0^{\circ}$ after surgery.

\section{Change in LL by Patient Positioning}

The average LL angles in standing, supine, and prone positions were $7.8^{\circ}, 22.1^{\circ}$, and $22.0^{\circ}$, respectively. The change in LL increased significantly by change in position from standing to supine or prone $(\mathrm{p}<0.001)$. Prone LL was approximately equal to supine LL (Fig. 2). In 80 of 85 cases (94.1\%), the difference between supine LL and prone LL was within $5^{\circ}$ (Fig. 3).

\section{Comparison Between Preoperative Severe and Mild Deformity Groups}

There were 60 patients in the severe deformity group (PI-LL $>30^{\circ}$ ) and 25 patients in the mild deformity group $\left(\mathrm{PI}-\mathrm{LL} \leq 30^{\circ}\right.$ ). The change in LL according to each position in the two groups is summarized in Table 2.

In the severe deformity group, standing LL, supine LL, and prone LL were $-0.5^{\circ}, 18.1^{\circ}$, and $18.3^{\circ}$, respectively.

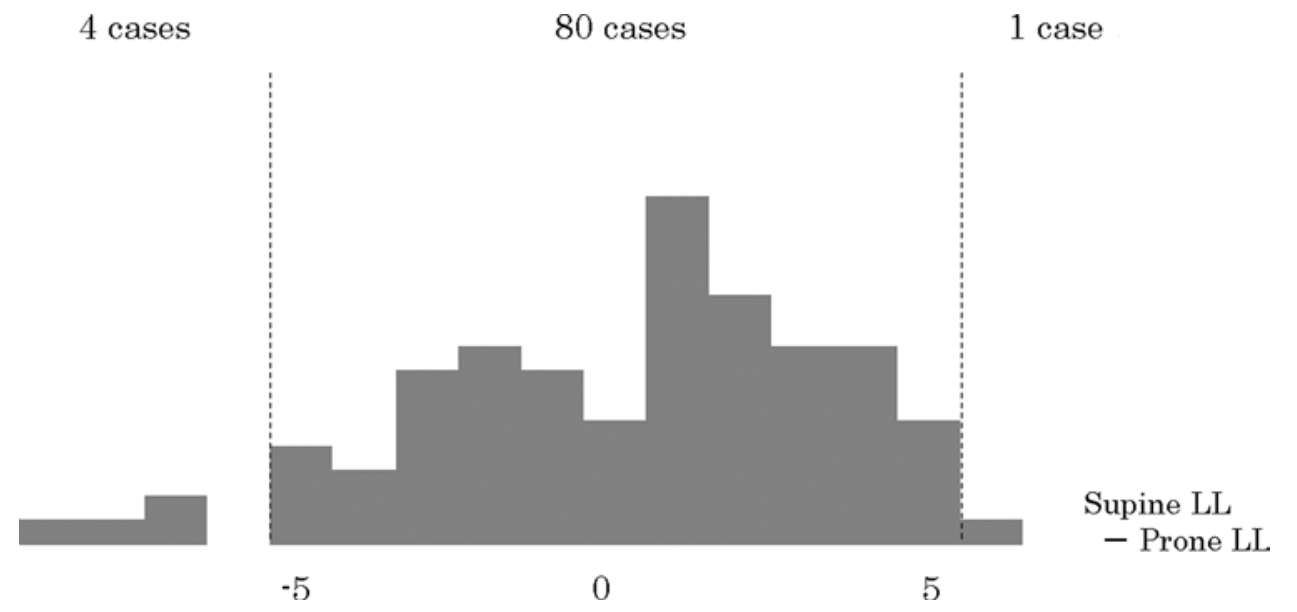

FIG. 3. Distribution of the difference between supine LL and prone LL (supine LL minus prone LL). In 80 of 85 patients ( $94.1 \%$ ), the difference between supine LL and prone LL was within $5^{\circ}$. 
TABLE 2. LL in standing, supine, and prone positions

\begin{tabular}{lrccc}
\hline \multicolumn{1}{c}{ Position } & All Patients & Severe Deformity $(\mathrm{PI}-\mathrm{LL}>30)$ & Mild Deformity $(\mathrm{PI}-\mathrm{LL} \leq 30)$ & $\mathrm{p}$ Value (severe vs mild) \\
\hline Standing LL $\left(^{\circ}\right)$ & $7.8 \pm 23.0$ & $-0.5 \pm 21.1$ & $27.8 \pm 13.4$ & $<0.001$ \\
\hline Supine LL $\left(^{\circ}\right)$ & $22.1 \pm 17.0$ & $18.1 \pm 16.3$ & $32.0 \pm 14.5$ & 0.002 \\
\hline Prone LL $\left(^{\circ}\right)$ & $22.0 \pm 15.8$ & $18.3 \pm 15.0$ & $30.9 \pm 14.2$ & 0.004 \\
\hline Change in LL & & & $4.2 \pm 9.3$ & $<0.001$ \\
\hline Standing to supine $\left(^{\circ}\right)$ & $14.3 \pm 15.1$ & $18.5 \pm 15.1$ & $3.1 \pm 8.8$ & $<0.001$ \\
\hline Standing to prone $\left(^{\circ}\right)$ & $14.2 \pm 15.2$ & $18.8 \pm 15.0$ & $1.1 \pm 3.0$ & 0.11 \\
\hline Supine to prone $\left(^{\circ}\right)$ & $-0.1 \pm 3.3$ & $-0.3 \pm 3.4$ & & \\
\hline
\end{tabular}

The changes in LL from standing to supine and prone positions were $18.5^{\circ}$ and $18.8^{\circ}$, respectively. In the mild deformity group, standing LL, supine LL, and prone LL were $27.8^{\circ}, 32.0^{\circ}$, and $30.9^{\circ}$, respectively. The changes in LL from standing to supine and prone positions were $4.2^{\circ}$ and $3.1^{\circ}$, respectively. The changes in LL from standing to supine and prone positions in the severe deformity group were significantly greater than those in the mild deformity group $(\mathrm{p}<0.001)$.

\section{Comparison Between Each Etiology}

The change in LL according to each position by each etiology is summarized in Table 3. The lordotic effect of change to supine and prone position in adult scoliosis was very small. The cases of neuromuscular deformity had the most lordotic effect in change to supine and prone position. However, LL in the supine position was approximately the same as that in the prone position in any etiology.

\section{Discussion}

This study investigated the impact of position on LL in patients with ASD. The lordotic effect of the intraoperative prone position was remarkable in patients with severe deformities. Furthermore, LL in the supine position was approximately equal to $L L$ in the prone position.

Previous studies concerning the impact of prone positioning on LL are summarized in Table 4. ${ }^{3,5,8-10,14,15}$ All the studies, except those by Fei et al. and Harimaya et al., investigated patients who underwent spinal surgery for degenerative lumbar disorders and not for deformities. In degenerative diseases, LL in the prone position had decreased or slightly increased compared with that seen in the standing position. Otherwise, LL in the prone position increased significantly compared with that in the standing position in our patients with ASD. In particular, in the severe deformity group, the lordotic effect of the intraoperative prone position was remarkable. Thus, it is important to evaluate and understand spinal alignment in not only the standing position but also the intraoperative prone position.

Harimaya et al. and Fei et al. reported changes in LL in patients with spinal deformity in upright, supine, and prone positions. ${ }^{3,5} \mathrm{LL}$ angles in the upright, supine, and intraoperative prone positions were $38.1^{\circ}, 46.0^{\circ}$, and $46.2^{\circ}$ in Harimaya's report and $23.5^{\circ}, 25.5^{\circ}$, and $24.6^{\circ}$ in Fei's report, respectively. Supine LL was almost the same as prone LL in both reports. In $94.1 \%$ of our patients, the difference between supine LL and prone LL was within $5^{\circ}$. Thus, intraoperative prone LL could be approximated by preoperative supine LL. Therefore, we believe that the assessment of preoperative supine lateral lumbar radiographs will help in the planning of corrective spinal surgeries in patients with ASD.

Several limitations of this study should be acknowledged. First, this study included various etiologies of spinal deformity. Although lumbar flexibility may differ depending on etiology, this result will be applicable in various etiologies. However, a larger sample size is required since the sample size was too small to analyze in more detail by each etiology. Additionally, the apex of the deformity also varied. Although in most cases the main deformity was in the lumbar region, it was difficult to determine the apex of deformity in flat lumbar and whole kyphosis cases. However, one advantage of this study was that LL in the prone position was found to be the same as LL in the supine position in various ASD

TABLE 3. LL in standing, supine, and prone positions by each etiology

\begin{tabular}{lccccc}
\hline & Kyphoscoliosis & Kyphosis & Vertebral Fracture & Neuromuscular & Adult Scoliosis \\
\hline No. of patients & 44 & 19 & 9 & 10 & 3 \\
\hline Standing LL $\left(^{\circ}\right)$ & $11.2 \pm 22.1$ & $5.1 \pm 15.4$ & $9.1 \pm 24.3$ & $-8.2 \pm 32.8$ & $27.3 \pm 15.3$ \\
\hline Supine LL $\left(^{\circ}\right)$ & $25.5 \pm 15.0$ & $20.3 \pm 9.3$ & $21.2 \pm 23.5$ & $10.1 \pm 24.9$ & $27.0 \pm 19.5$ \\
\hline Prone LL $\left(^{\circ}\right)$ & $25.2 \pm 14.2$ & $19.7 \pm 9.7$ & $21.2 \pm 20.7$ & $10.9 \pm 22.4$ & $28.7 \pm 18.6$ \\
\hline Change in LL & & & & & $-0.3 \pm 5.9$ \\
\hline Standing to supine $\left(^{\circ}\right)$ & $15.2 \pm 13.0$ & $15.3 \pm 13.0$ & $12.1 \pm 22.3$ & $18.3 \pm 22.5$ & $1.3 \pm 3.8$ \\
\hline Standing to prone $\left(^{\circ}\right)$ & $14.2 \pm 12.8$ & $14.6 \pm 13.5$ & $12.1 \pm 21.3$ & $19.1 \pm 22.7$ & $1.6 \pm 2.5$ \\
\hline$\quad$ Supine to prone $\left(^{\circ}\right)$ & $-0.3 \pm 3.4$ & $-0.6 \pm 3.1$ & $0.0 \pm 3.3$ & $0.8 \pm 3.9$ & \\
\hline
\end{tabular}


TABLE 4. LL in standing and prone positions in previous reports

\begin{tabular}{lccc}
\hline \multicolumn{1}{c}{ Authors \& Year } & $\begin{array}{c}\text { Average Age } \\
(\mathrm{yrs})\end{array}$ & $\begin{array}{c}\text { Standing LL } \\
\left({ }^{\circ}\right)\end{array}$ & $\begin{array}{c}\text { Prone LL } \\
\left({ }^{\circ}\right)\end{array}$ \\
\hline Tan et al., 1994 & 35.7 & 55.6 & 28.3 \\
\hline Peterson et al., 1995 & 46.5 & 61.7 & 62.8 \\
\hline Tribus et al., 1999 & NR & 51 & 37 \\
\hline Lee et al., 2008 & 58.3 & 48.1 & 39 \\
\hline Harimaya et al., 2009 & 57.4 & 38.1 & 46.2 \\
\hline Lee et al., 2016 & 67.8 & 43.5 & 48.8 \\
\hline Fei et al., 2017 & 63.8 & 23.5 & 24.6 \\
\hline Present series & 70.2 & 7.8 & 22 \\
\hline Severe deformity & 70.7 & -0.5 & 18.8 \\
\hline Mild deformity & 69.2 & 27.8 & 30.9 \\
\hline
\end{tabular}

$\mathrm{NR}=$ not reported.

cases. Furthermore, although different spinal frames might affect LL in the prone position, we only evaluated one type of frame, and validation using various frames will be required.

\section{Conclusions}

The lordotic effect of the intraoperative prone position was greater in patients with severe deformities. LL in the prone position was approximately equal to LL in the supine position; in 80 of 85 cases $(94.1 \%)$ the difference was within $5^{\circ}$. Therefore, assessment of preoperative supine lateral lumbar radiographs will help in the planning of corrective spinal surgeries in patients with ASD.

\section{References}

1. Dorward IG, Lenke LG: Osteotomies in the posterior-only treatment of complex adult spinal deformity: a comparative review. Neurosurg Focus 28(3):E4, 2010

2. Enercan M, Ozturk C, Kahraman S, Sarıer M, Hamzaoglu A, Alanay A: Osteotomies/spinal column resections in adult deformity. Eur Spine J 22 (Suppl 2):S254-S264, 2013

3. Fei H, Li WS, Sun ZR, Jiang S, Chen ZQ: Effect of patient position on the lordosis and scoliosis of patients with degenerative lumbar scoliosis. Medicine (Baltimore) 96:e7648, 2017

4. Glassman SD, Bridwell K, Dimar JR, Horton W, Berven S, Schwab F: The impact of positive sagittal balance in adult spinal deformity. Spine (Phila Pa 1976) 30:2024-2029, 2005

5. Harimaya K, Lenke LG, Mishiro T, Bridwell KH, Koester LA, Sides BA: Increasing lumbar lordosis of adult spinal deformity patients via intraoperative prone positioning. Spine (Phila Pa 1976) 34:2406-2412, 2009

6. Horton WC, Brown CW, Bridwell KH, Glassman SD, Suk SI, Cha CW: Is there an optimal patient stance for obtaining a lateral 36" radiograph? A critical comparison of three techniques. Spine (Phila Pa 1976) 30:427-433, 2005

7. Lafage V, Schwab F, Patel A, Hawkinson N, Farcy JP: Pelvic tilt and truncal inclination: two key radiographic parameters in the setting of adults with spinal deformity. Spine (Phila Pa 1976) 34:E599-E606, 2009

8. Lee JH, Lee JH, Yoon KS, Kang SB, Jo CH: Effect of intraoperative position used in posterior lumbar interbody fusion on the maintenance of lumbar lordosis. J Neurosurg Spine 8:263-270, 2008

9. Lee SK, Lee SH, Song KS, Park BM, Lim SY, Jang G, et al: Lumbar lordosis of spinal stenosis patients during intraoperative prone positioning. Clin Orthop Surg 8:65-70, 2016

10. Peterson MD, Nelson LM, McManus AC, Jackson RP: The effect of operative position on lumbar lordosis. A radiographic study of patients under anesthesia in the prone and 90-90 positions. Spine (Phila Pa 1976) 20:1419-1424, 1995

11. Rose PS, Bridwell KH, Lenke LG, Cronen GA, Mulconrey DS, Buchowski JM, et al: Role of pelvic incidence, thoracic kyphosis, and patient factors on sagittal plane correction following pedicle subtraction osteotomy. Spine (Phila Pa 1976) 34:785-791, 2009

12. Schwab F, Lafage V, Patel A, Farcy JP: Sagittal plane considerations and the pelvis in the adult patient. Spine (Phila Pa 1976) 34:1828-1833, 2009

13. Schwab F, Ungar B, Blondel B, Buchowski J, Coe J, Deinlein D, et al: Scoliosis Research Society-Schwab adult spinal deformity classification: a validation study. Spine (Phila Pa 1976) 37:1077-1082, 2012

14. Tan SB, Kozak JA, Dickson JH, Nalty TJ: Effect of operative position on sagittal alignment of the lumbar spine. Spine (Phila Pa 1976) 19:314-318, 1994

15. Tribus CB, Belanger TA, Zdeblick TA: The effect of operative position and short-segment fusion on maintenance of sagittal alignment of the lumbar spine. Spine (Phila Pa 1976) 24:58-61, 1999

16. Yamato Y, Hasegawa T, Kobayashi S, Yasuda T, Togawa D, Arima H, et al: Calculation of the target lumbar lordosis angle for restoring an optimal pelvic tilt in elderly patients with adult spinal deformity. Spine (Phila Pa 1976) 41:E211-E217, 2016

\section{Disclosures}

Daisuke Togawa and Shin Oe belong to the Division of Geriatric Musculoskeletal Health, a laboratory funded by Medtronic Sofamor Danek Inc., Japan Medical Dynamic Marketing Inc., and Meitoku Medical Institution Jyuzen Memorial Hospital. Daisuke Togawa is the deputy editor of the Journal of Bone and Joint Surgery.

\section{Author Contributions}

Conception and design: Yasuda, Hasegawa, Yamato, Togawa, Kobayashi, Matsuyama. Acquisition of data: all authors. Analysis and interpretation of data: all authors. Drafting the article: Yasuda. Critically revising the article: Yasuda. Reviewed submitted version of manuscript: all authors. Approved the final version of the manuscript on behalf of all authors: Yasuda. Statistical analysis: Yasuda. Administrative/technical/material support: Yasuda. Study supervision: Matsuyama.

\section{Correspondence}

Tatsuya Yasuda: Hamamatsu Medical Center, Hamamatsu, Japan. t.yasuda0820@gmail.com. 